\title{
Design and Preliminary Affective Characterization of a Novel Fabric-based Tactile Display
}

\author{
M. Bianchi ${ }^{+\diamond *}$ \\ G. Valenza ${ }^{\diamond} \quad$ A. Serio $\diamond \ddagger$ \\ E.P. Scilingo ${ }^{\diamond * *}$ \\ A. Lanatà $§$ \\ A. Greco»II \\ M. Nardelli»\| \\ A. Bicchi ${ }^{\diamond+\dagger \dagger}$ \\ + Department of Advanced Robotics, Istituto Italiano di Tecnologia, via Morego, 30, 16163 Genova, Italy. \\ ${ }^{\diamond}$ Research Center "Enrico Piaggio", University of Pisa, Largo L. Lazzarino, 1, 56126 Pisa, Italy.
}

\begin{abstract}
In this work we present a novel wearable haptic system based on an elastic fabric which can be moved forward and backward over the user forearm thus simulating a human caress. The system allows to control both the velocity of the "caress-like" movement, by regulating motor velocity, and the "strength of the caress", by regulating motor positions and hence the force exerted by the fabric on the user forearm. Along with a description of the mechanical design and control of the system, we also report the preliminary results of psycho-physiological assessment tests performed by six healthy participants. Such an assessment is intended as a preliminary characterization of the device capability of eliciting tactually emotional states in humans using different combinations of velocity and caress strength. The emotional state is expressed in terms of arousal and valence. Moreover, the activation of the autonomic nervous system is also evaluated through the analysis of the electrodermal response (EDR). The main results reveal a statistically significant correlation between the perceived arousal level and the "strength of the caress" and between the perceived valence level and the "velocity of the caress". Moreover, we found that phasic EDR is able to discern between pleasant and unpleasant stimuli. These preliminary results are very encouraging and confirm the effectiveness of this device in conveying emotional-like haptic stimuli in a controllable and wearable fashion.
\end{abstract}

Index Terms: H.5.2 [Information Interfaces and Presentation (e.g., HCI)]: User Interfaces-Haptic I/O; H.1.2 [Information Systems]: User/Machine Systems-Human Factors; I.5.4 [Pattern Recognition]: Applications-Signal Processing; J.4 [Social and Behavioral Sciences]: Psychology-.

\section{INTRODUCTION}

In the recent years the investigation on the role of haptic cues in evoking human emotions has gained an increasing attention, since touch represents one of the most ancestral human senses [1], highly emotionally charged [13]. Affective reactions and emotional response elicited by haptic stimuli and interactions were investigated e.g. in $[15,23,21,12,24]$. Considering synthetic and artificial tactile stimuli, devices and haptic systems that can elicit, enhance, or influence the user emotional state were also developed and studied

\footnotetext{
*e-mail: m.bianchi@iit.it

†e-mail: g.valenza@ieee.org

† e-mail: alessandro.serio@centropiaggio.unipi.it

§e-mail: a.lanata@centropiaggio.unipi.it

Ile-mail: alberto.greco@centropiaggio.unipi.it

॥e-mail: nardemi@gmail.com

**e-mail: e.scilingo@centropiaggio.unipi.it

†ेe-mail: bicchi@centropiaggio.unipi.it
}

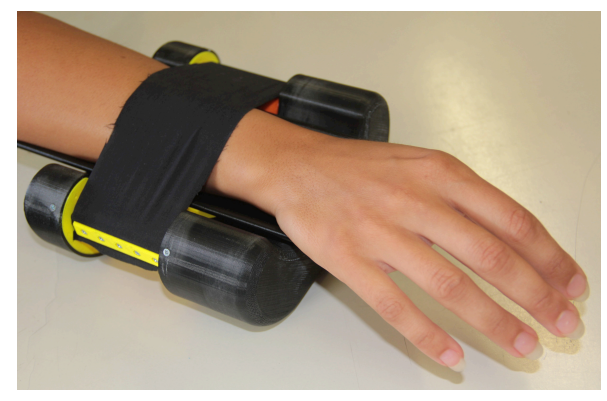

Figure 1: An overview of the haptic system worn by one of the participants during the experimental tests.

e.g. in $[25,14]$. In [31], the authors presented the "Haptic Creature Project", whose goal was to understand affect display through touch, during human-robot social interaction. To achieve such objective, they developed the "Haptic Creature", a robot that mimics a small pet that interacts through touch. The effectiveness of this robot in communicating its state of arousal was demonstrated in [30] but less so for valence.

In this paper we present a novel wearable haptic system (see fig. 1 ), which is based on an elastic fabric connected to two motors and which can move over the user forearm to simulate a human caress. Notice that tactile displays that used an elastic fabric to convey stimuli to the user were presented in $[5,6,22]$. However, these displays significantly differ from the here proposed device under several aspects. First, they were not wearable. Second, they were used for softness rendering and were thought to be able to interact with the human fingertip, which "actively" pressed against their fabric surface. The fabric stretching state was then modulated to convey softness cues, according to the contact area information [7] that was optically measured. On the contrary the device we describe in this paper was thought to be worn by the user, in order to convey to her/his forearm force and velocity information for simulating a human caress. The system can regulate the velocity of the "caress-like" movement, by controlling motor velocity, and the "strength of the caress", by controlling motor positions and hence the force exerted on the user forearm (which depends on how much the fabric is wrapped around the forearm). After the description of the system, we report the results of a preliminary affective characterization with healthy participants. We asked participants to report their emotional response to the stimuli conveyed by the device. For this reason, we adopted a common dimensional model, which uses multiple dimensions to categorize emotions, the Circumplex Model of Affect (CMA) [18, 28, 27, 26]. The CMA allows to take into account two main dimensions conceptualized by the terms of valence and arousal (see fig. 2). According to [18], all affective states arise from two fundamental neurophysiological systems, one related to valence (pleasure/displeasure) and the other to arousal, or alertness; 


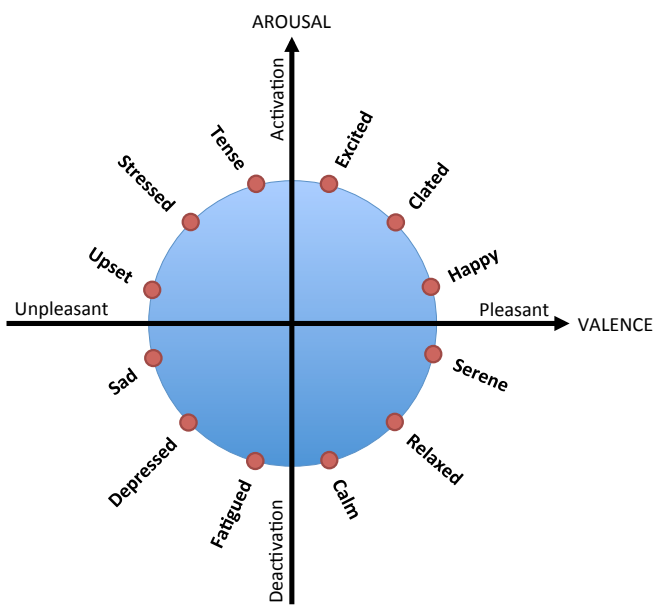

Figure 2: The Circumplex Model of Affect (CMA), with the two affective dimensions of arousal and valence.

or in other terms, as it was reported in [32], “...one dimension, valence, is described by the continuum from unpleasant to pleasant, while the other dimension, arousal, ranges from deactivated to activated". Moreover, the connection between the two dimensions (arousal and valence) and the autonomic nervous system response was showed in many studies such as [19].

Each combination of force and velocity values (i.e. "strength of the caress" and "velocity of the caress", respectively) was assessed by participants in terms of arousal and valence, considering five levels (including the neutral one).

At the same time, in order to verify if the tactile stimuli can also evoke a change in the patterns of the Autonomic Nervous System (ANS), we recorded the ElectroDermal Response (EDR) dynamics of the participants. The rationale behind this choice was justified by the fact that EDR is directly related to the Sympathetic Nervous System (SNS) [8], whose dynamics is strongly affected by emotional changes (see e.g. [11, 16, 28, 27, 26, 29] for detailed reviews and further information). Moreover, EDR results to be also strongly related to mechanoreceptive myelinated fibers located under the skin and sympathetic changes are consistent with mechanoreceptor outcomes. More specifically, the sensitivity of cutaneous mechanoreceptors is mediated by a direct action of efferent neurotransmitters on the receptor membrane [10].

The main results from this preliminary study show a statistically significant correlation between the perceived arousal level and the "strength of the caress" and between the perceived valence level and the "velocity of the caress". Furthermore, EDR analysis also indicates that the mechanical skin stimulation produced by the here presented device is able to elicit ANS changes.

\section{Description of Tactile Display}

The device we developed uses a layer of elastic fabric to convey haptic stimuli. We selected the elastic Superbiflex by Mectex S.P.A for its high resistance to traction and the good elastic behavior exhibited in a large range of elasticity [5].

More specifically, the extremities of a rectangular-shaped fabric $(60 \mathrm{~mm} \times 160 \mathrm{~mm})$ were connected by means of screws to two rolls, each of them was independently moved by one motor (HITEC digital DC servomotor HS-7954 $\mathrm{H}$ with an input voltage of $7.4 \mathrm{~V}$ ). The choice of this kind of motors was motivated by the fact that they provide a good trade-off between velocity and torque. Furthermore, we suitably modified the motors to allow a continuous rotation of the motor shafts, which we directly connected to the rolls (see fig. 3 for further details). The motor positions and rotation velocity were

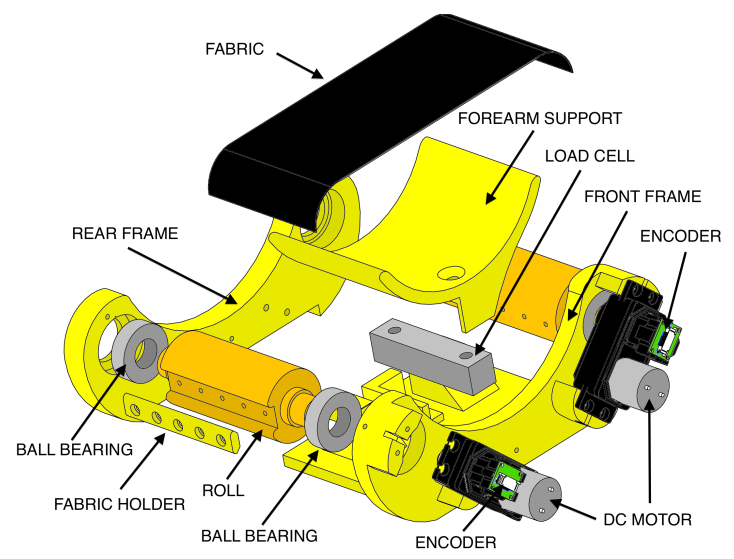

Figure 3: The system: exploded draw without the cover. The total dimensions of the system can be inscribed in a parallelepiped of dimensions $150 \times 150 \times 80 \mathrm{~mm}$.

controlled by a customized electronic board (PSoC-based electronic board with RS-485 communication protocol), which can read motor positions by using two absolute magnetic encoders (12 bit magnetic encoder by Austrian Microsystems AS5045 with a resolution of $0.0875^{\circ}$ ).

Finally, we endowed the system with a load cell (Micro Load Cell $[0-5 \mathrm{~kg}]$ with a resolution of $5 \mathrm{~g}$ by Phidgets) placed at the basis of the forearm support to record the normal force exerted by the fabric on the forearm. We realized the device using ABS plastic material and then encapsulated the system within a plastic cover to protect motors.

An exploded draw of the system is shown in fig. 3 .

\subsection{How the Tactile Display Works}

The system was designed to be wearable, as it can be applied to the user forearm without discomfort (see also fig. 1). The user must place her/his forearm on the forearm support (half hollow cylinder, radius $45 \mathrm{~mm}$ and length $60 \mathrm{~mm}$, see fig. 3), under the fabric. When the device is active, we can distinguish two different operating phases (see fig. 4):

Calibration Phase: in this phase we calibrate the force exerted by the fabric on the user forearm. There are two modalities of control: when motor 1 rotates in a counter-clockwise direction and motor 2 rotates in a clockwise direction, the fabric is increasingly wrapped around the forearm thus pressing it with an increasing force (as it is reported in fig. 4), which can be recorded by the load cell. Conversely, when motor 1 rotates in a clockwise direction and motor 2 in a counter-clockwise direction the force exerted by the fabric on the forearm decreases. In this manner it is possible to modulate the "strength of the caress" by suitably controlling the positions of the motors. The maximum level of force that can be applied is $20 \mathrm{~N}$.

Movement Phase: when we achieve the desired level of force exerted by the fabric and both motors are in the reference position, they start to coherently rotate and the fabric moves forward and backward over the user forearm, simulating a caress. The velocity of the caress can be modulated by regulating the velocity of the motors. More specifically, since the electronic board is endowed with a built-in motor position controller, we can feed the motors with a sinusoidal input reference trajectory, whose frequency and amplitude can be set to control the velocity and the amplitude of motor rotation, respectively. The maximum angular displacement of the motors from the reference positions is set to $\pm 90^{\circ}$. An entire control cycle lasts $1 \mathrm{~ms}$ and the fastest sinusoidal input (frequency of $5 \mathrm{~Hz}$ ) can be followed with a phase delay less than $2 \mathrm{~ms}$. 


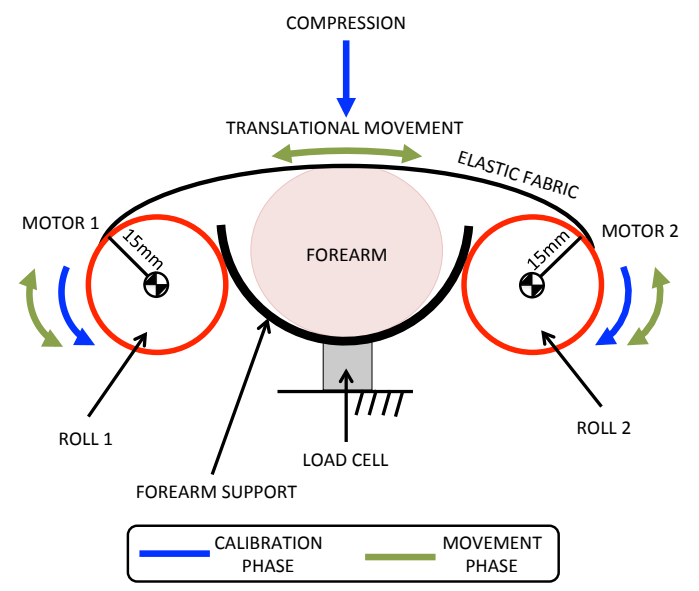

Figure 4: Front view: how the system interacts with the user forearm. The Movement Phase is displayed in green while the Calibration Phase is displayed in blue. In this case the level of force must increase. The radius of each roll is $15 \mathrm{~mm}$.

\section{Preliminary User Study}

In order to test and categorize the emotional response elicited by the stimuli conveyed by the system, we carried out several psychophysiological experiments with a set of haptic stimuli, by varying the force exerted by the fabric and its velocity over the forearm of the participants. The goal was to simulate different levels of "strength of the caress" and different levels of "velocity of the caress" and investigate their emotional impact on participants, using both self-assessment procedures and EDR recordings.

\subsection{Material and Methods}

We used 15 combinations among 5 levels of force exerted by the fabric $(0 \mathrm{~N}, 2 \mathrm{~N}, 4 \mathrm{~N}, 6 \mathrm{~N}, 8 \mathrm{~N})$, to simulate different levels of "strength of the caress", and 3 levels of velocity $(9.4 \mathrm{~mm} / \mathrm{s}, 37$ $\mathrm{mm} / \mathrm{s}, 65 \mathrm{~mm} / \mathrm{s}$ ), to simulate different "velocities of the caress". These velocity levels can be obtained by feeding the motors with four different sinusoidal input trajectories, whose frequencies are set to $0.1 \mathrm{~Hz}, 0.4 \mathrm{~Hz}, 0.7 \mathrm{~Hz}$, respectively, and can be computed by knowing the radius of the rolls $(15 \mathrm{~mm})$. The velocities we used are coherent with the ones reported in [12] and they reasonably represent an exhaustive range of the movements a human hand can do while is performing a caress. The forces we used are higher than the ones reported in [12] but they were heuristically chosen to simulate typical parameters of the human caress. When the force increases, the fabric is more closely wrapped around the forearm. This is the case of a caress performed with a non negligible force; when humans perform such a type of gesture, the hand wraps the forearm, the friction force increases and there is no more pure sliding (as with light forces) but also skin torsion. This behavior can be observed both in "real" and "artificial" caress and its investigation was coherent with the aim of the paper, i.e. trying to reproduce as exhaustively as possible the behavior of the human caress. The range of forces was preliminarily selected in an informal piloting conducted prior to the study in order to not convey painful stimuli to participants. Notice that when the force was $0 \mathrm{~N}$, the fabric was only lightly in contact with the forearm.

\subsection{Participants and Experimental Setup}

Six right-handed participants (4 male (M) and 2 female (F), with average age of $28.8 \pm 5.9)$ gave their informed consent to participate in the study. No participants reported physical limitations and any experience of mental or personality disorder in their life, that would affect the experimental outcomes. This study was approved by the Ethical Committee of the University of Pisa .

Participants were comfortably seated, the right forearm was horizontal and placed on the forearm support, hand palm down. For all trials, participants wore in-ear headphones in order to prevent any auditory cues. Prior to the experiment, the load cell was auto calibrated with respect to the forearm weight.

The experiment consisted of two phases. The first phase, named resting phase, lasted 5 minutes and aimed at detecting the EDR baseline. During this phase participants were seated on a comfortable chair, keeping their eyes closed. At the end of this phase, the stimuli were conveyed to participants. During the Calibration Phase, we controlled motor positions to achieve the desired level of pressing force on the forearm. Afterwards, in the Movement Phase, the fabric moved over the forearm with the desired velocity. In the Movement Phase, the motors were coherently moved once in a clockwise and once in a counterclockwise manner, without delay between the two rotations, by imposing an angular displacement of $\mp 90^{\circ}$ (rotations are assumed to be positive if they occur in the counter-clockwise direction). Each stimulus consisted of a combination of force and velocity. All the combinations were suitably randomized and repeated three times for each participant, with an inter-stimulus interval of 15 seconds and stimulus duration of $7 \mathrm{~s}$, for a total number of trials per participant of 45 . The choice of the duration of the stimulus was justified by the fact that the prefrontal cortex represents one of the core central pathway of cognitive sensation: affective elicitation longer than 6 seconds allows the prefrontal cortex to encode the stimulus information and transmit it to other areas of the Central Autonomic Network down to the brainstem, thus producing a context appropriate response [20]. Accordingly, it is reasonable to hypothesize that EDR changes are representative of affective haptic stimuli lasting for more than 6 seconds. In order to limit the impact of the novelty of the device on the experimental results, prior to the experiment participants were trained briefly and allowed to experience some selected stimuli. In any case such aspect will be investigated more in depth as future work.

We asked participants to report their emotional response to the stimuli conveyed by the device. After each stimulus, participants were invited to indicate the level of arousal and valence through the Self Assessment Manikin (SAM) [9]. SAM is a pictorial assessment technique that directly measures the pleasure (valence) and arousal associated with a person affective reaction to stimuli. More specifically, w.r.t the valence, participants were invited to indicate the picture of the SAM, whose dimension better described how much, from unpleasant to pleasant, was the feeling arisen by the experienced stimulation. For the arousal, participants were invited to indicate the picture of the SAM, whose dimension better described how much, from deactivated to activated, was the feeling arisen by the experienced stimulation. The choice to use SAM evaluation scale, which requires only two simple judgments, was in agreement with [9], where the authors showed a strong correlation between SAM evaluation scale and the Semantic Differential Scale [17] with 18 different ratings, both for experienced pleasure and felt arousal. More specifically, the SAM we used for our experiments consisted of 10 items, 5 levels of arousal (i.e. from 1 to 5 , in an increasing scale, ranging from neutral to emotionally strong) and 5 levels for the valence (i.e. from -2 to 2 , in a scale from highly unpleasant stimuli to highly pleasant stimuli, neutral level 0 ).

At the same time, for the whole experimental session, we acquired the EDR signal at the index and middle fingertips of participant non-dominant hand. The EDR signal was recorded by using the BIOPAC MP150 with the EDR biosensor. The sampling rate was $500 \mathrm{~Hz}$. We increased the Signal-to-Noise ratio by means of a low-pass filter, which limited the frequency bandwidth in the range from 0 to $2 \mathrm{~Hz}$. The entire session lasted 18 minutes for each participant. Notice that we did not record physiological data to in- 


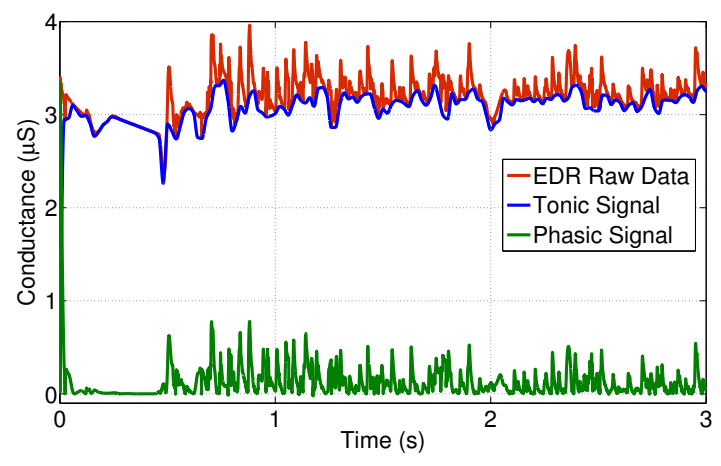

Figure 5: An exemplary plot showing the phasic and tonic components of an acquired EDR signal.

dividuate a second baseline at the end of the study. Indeed, since we dealt with non-invasive emotional elicitation and recordings, it is reasonable to hypothesize that there are no longer-term changes in the EDR dynamics due to caress-like stimuli. However, we will demand the investigation of such a possible effect as future work.

\subsection{Methodology of EDR Acquisition and Signal Processing}

The EDR signal is generated by the eccrine sweat glands activity, whose control is performed by the central nervous system through the SudoMotor Nerve Activity (SMNA). Such an activity leads to changes on the skin impedance, which can be measured through several approaches. In this work, we adopted a small continuous voltage applied to the skin to induce the current that was measured through two standard $\mathrm{Ag} / \mathrm{AgCl}$ electrodes positioned at the index and middle fingertips of the non-dominant hand. The ratio between the voltage drop and the induced current represents the skin electric impedance, i.e. the EDR.

From a signal processing point of view, the EDR signals, whose changes depend on the individual physiological state as well as on the interaction with environmental events, can be characterized by a slowly-varying component, the so-called tonic component, and a superposed phasic component, which has a short rise time followed by a slower recovery time. The tonic component represents the baseline level of the skin impedance, whose trend is different from person to person and depends on both physiological state and autonomic regulation. The phasic component varies with specific external stimuli such as lights, sounds, smells, or events and it is defined as the part of the signal which arises within a predefined response window (1 - $5 \mathrm{~s}$ after stimulus onset), satisfying a minimum amplitude criterion $(0.05 \mu S)$ [4].

In order to effectively split the raw EDR signal into tonic and phasic components, we used the so-called "deconvolutive" approach of analysis [3]. For sake of comprehension, an exemplary plot showing the phasic and tonic EDR acquired during the experimental session is shown in fig. 5 .

We extracted several features from both the tonic and phasic components, within non-overlapped time windows of 6 seconds, synchronized with the beginning of the haptic stimulus. Each feature was normalized by subtracting the value it assumed in the resting condition. More specifically, we calculated the mean value (MEAN), the maximum value (MAX) and the area under the phasic and tonic driver curve (AUC). The MAX feature indicates the maximum value of the sympathetic activation, whereas the MEAN and the AUC explain the number and the strength of sympathetic sudomotor nerve discharges [2], respectively. Finally, we computed also the standard deviation (STD) of both driver signals as measures of data dispersion.

\subsection{Statistical analysis}

According to other affective studies presented in literature [33, 12], we report the self-reported scores averaged over participants, assuming stimuli as a set of statistically independent samples. Intrasubject variability effect will be analyzed as future work.

Statistical analyses comprise non-parametric Spearman correlation and Kruskal-Wallis (KW) test. The choice of non-parametric rank-based statistics was justified by the intrinsically discrete nature of the force and velocity levels of stimuli as well as of the self-reported arousal and valence scores. Moreover, the feature set extracted from the EDR signals was tested by means of Lilliefors test and resulted to be non-gaussian distributed. The significance level $\alpha$ was set to 0.05 .

\section{Results and Discussion}

We performed the correlation analysis between the self-reported arousal and valence scores and "the strength and the velocity of the caress". We found a positive correlation between the arousal scores and the "strength of the caress" $\left(\rho=0.68, p<10^{-6}\right.$ with the null hypothesis of having no correlation between samples). This result is encouraging and leads us to suppose that participants perceived an increasing arousing tactile stimulus as the pressing brushing force against the arm grown. At the same time, we found a small but significant correlation between valence and force $(\rho=-0.16$; $p<0.01)$. Such a result slightly suggests that the pleasantness of tactile stimuli is inversely related to the strength. However, this finding must be further investigated.

We found a negative correlation coefficient $(\rho=-0.32, p<$ $10^{-6}$ ) between valence scores and the "velocity of the caress", under the same null hypothesis of having no correlation between samples. It means that slower velocities of the mimicked caress are more likely associated to a pleasant stimulus. This result is coherent with the trend observed for grating stimuli on the forearm in [12], for the increase in velocity to reduce tactile pleasantness. This result provides important cues, to be deeply investigated further, on the inverse relationship between the caress velocity and the pleasantness of the tactile perception. On the contrary, we found no statistically significant correlation between arousal and velocity levels $(\rho=0.0051 ; p>0.05)$.

Concerning the statistical analysis, the KW test reveals no significant difference in the arousal scores among the 3 levels of velocity $(p>0.05)$, whereas significant differences in the arousal scores were found among the 5 levels of force with $p<10^{-6}$. This means that at least one level is different from the others. After a post-hoc analysis with Bonferroni correction, we found no differences between force levels 1 and 2 and between 2 and 3 but significative differences arose when comparing all the other force levels each other $(p=0.019$ between level 4 and $5, p=0.005$ between level 1 and $3, p<10^{-6}$ for all the other pairs). According to this result, we decided to remove from the statistical analysis the level of force 2; as expected, in this case all the 4 levels of force $(1,3,4$ and 5) produced statistically different arousing emotions. This outcome is very promising for further developments of the device and for the design of future experiments. Likewise, the KW test reveals no significant difference in the valence scores among the 5 levels of force $(p>0.05)$, whereas significant differences in the valence scores were found among the 3 levels of velocity with $p<10^{-6}$. Post-hoc analysis shows a significant difference between velocity levels 1 vs 2 and 1 vs. $3\left(p<10^{-4}\right)$. Even in this case we can conclude that velocities 2 and 3 are affectively perceived with the same level of pleasantness w.r.t the level 1. Finally, we also evaluated the interaction effects between velocity and force for both arousal and valence dimensions. Results reveal no statistical significance for the interaction term $(p>0.05)$.

We analyzed the EDR signal in order to find specific features that were able to assess the self-reported emotions. We applied 


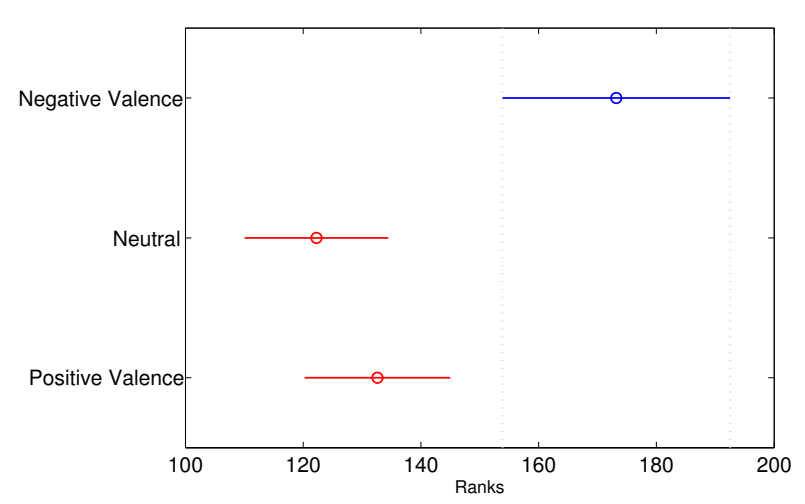

Figure 6: The statistical results of the post-hoc tests on the valence levels (on the $y$-axis), for the maximum values of the phasic components of EDR signals. The neutral level (Neutral) is not distinguishable from the pleasant category (Positive Valence), while the pleasant category and the neutral one are distinguishable from the negative category (Negative Valence).

the KW test to the extracted features and we found no significant differences among the arousal levels. On the contrary, for the valence, putting together the levels -2 and -1 into a generic unpleasant category and levels 1 and 2 into a pleasant category, and leaving the level 0 as neutral, we found that the maximum value (MAX) of the phasic component, calculated within the 6 seconds lasting window after the onset of the stimulus, enables to statistically discriminate the two categories of pleasant and unpleasant stimuli with $p<10^{-3}$ (Bonferroni correction). The neutral level is not distinguishable from the pleasant category, whereas it is distinguishable from the unpleasant category; in other terms, the phasic EDR is not able to finely discern all the valence levels, but rather it has statistical significance with only 3 levels. In fig. 6 we report the plot of the statistical test output. This result, although encouraging, needs to be further assessed with more dedicated experiments. Indeed, it is expected that EDR can be related to both arousal and valence, the latter being more challenging to be communicated, see e.g. [30]. In any case, the main finding from the EDR analysis is that the mechanical skin stimulation produced by the device seems to be able to elicit ANS changes. Finally, we would like to underline that it is a common practice in psycho-physiology to merge some of the valence and arousal levels in order to simplify further analyses $[11,26]$. Indeed, it may happen that a specific physiological correlate, as the phasic EDR feature, is not able to finely discern each of the levels of valence (arousal) but rather it has statistical power for grouped levels only. However we will re-run the study on a separate participant pool with the modified values as future work, in order to investigate more in depth the experimental outcomes and to draw stronger conclusions.

\section{Conclusion ANd Future Work}

In this paper we have presented a novel wearable device able to elicit standardized affective caress-like haptic stimuli. Such a device was preliminarily characterized in terms of perceived arousal and valence levels through self assessment reports and EDR recordings.

Despite the inter-subject variability, we found significant correlations and statistical differences between the perceived arousal level and the "strength of the caress" and between the perceived valence level and the "velocity of the caress". Furthermore, these preliminary results suggest that the phasic component of the EDR signal is statistically different during the perceived pleasant and unpleasant stimuli. These results are in line with the theory proposed by
Calof et al. [10] that indicates that the mechanoreceptors are sensitive both to the pressure and sliding of the skin. Moreover it is worthwhile noticing that mechanoreceptor responses are mediated by the SMNA activity. Future work will aim to increase the number of participants with more dedicated experiments as well as to investigate the intra-subject and inter-trial variability of the elicited affective stimuli. Sex differences will be also considered [12] and we will analyze more in depth a wider number of extracted features of the EDR signal. The results presented in this manuscript, although preliminary, demonstrate the effectiveness of our wearable device in conveying emotional-like haptic stimuli and they might open interesting opportunities for the usage of such a haptic system.

One possible challenging application might be in clinical rehabilitative scenarios, where it is crucial to have wearable devices able to convey repeatable and controllable haptic stimuli. For example our haptic system might be profitably employed with patients with Disorders Of Consciousness (DOC), where standardized tactile stimuli could evoke a response in the autonomic nervous system. For this reason it will be important to further increase the wearability of the system in terms of portability and usability for long-term monitoring; to achieve this goal, the EDR signal might be monitored through a textile-based sensing glove [16].

\section{ACKNOWLEDGEMENTS}

This work is supported by the European Research Council under the ERC Advanced Grant no. 291166 SoftHands (A Theory of Soft Synergies for a New Generation of Artificial Hands). This work has received funding from the EU FP7/2007-2013 project no. 601165 WEARHAP (WEARable HAPtics for Humans and Robots) and project no. 248587 THE (The Hand Embodied).

\section{REFERENCES}

[1] Aristotle. De Anima. IV Century B.C.

[2] D. R. Bach, K. J. Friston, and R. J. Dolan. Analytic measures for quantification of arousal from spontaneous skin conductance fluctuations. International Journal of Psychophysiology, 76(1):52-55, 2010.

[3] M. Benedek and C. Kaernbach. A continuous measure of phasic electrodermal activity. Journal of neuroscience methods, 190(1):80-91, 2010.

[4] M. Benedek and C. Kaernbach. Decomposition of skin conductance data by means of nonnegative deconvolution. Psychophysiology, 47(4):647-658, 2010.

[5] M. Bianchi, E. P. Scilingo, A. Serio, and A. Bicchi. A new softness display based on bi-elastic fabric. World Haptics Conference, pages 382-383, 2009.

[6] M. Bianchi, A. Serio, E. P. Scilingo, and A. Bicchi. A new fabricbased softness display. In Proc. IEEE Haptics Symposium, pages 105 $-112,2010$.

[7] A. Bicchi, D. E. De Rossi, and E. P. Scilingo. The role of the contact area spread rate in haptic discrimination of softness. IEEE trans. on Robotics and Automation, 16(5):496-504, October 2000.

[8] W. Boucsein. Electrodermal acitivity. 1992.

[9] M. M. Bradley and P. J. Lang. Measuring emotion: the self-assessment manikin and the semantic differential. Journal of behavior therapy and experimental psychiatry, 25(1):49-59, 1994.

[10] A. Calof, R. Jones, and W. Roberts. Sympathetic modulation of mechanoreceptor sensitivity in frog skin. The Journal of physiology, 310(1):481-499, 1981.

[11] R. Calvo and S. D'Mello. Affect detection: An interdisciplinary review of models, methods, and their applications. Affective Computing, IEEE Transactions on, 1(1):18-37, 2010.

[12] G. K. Essick, F. McGlone, C. Dancer, D. Fabricant, and Y. Ragin. Quantitative assessment of pleasant touch. Neuroscience and Biobehavioral Reviews, 34(2):192-203, 2010.

[13] T. Field. Touch. MIT press, 2003. 
[14] E. Gatti, G. Caruso, M. Bordegoni, and C. Spence. Can the feel of the haptic interaction modify a user's emotional state? In In Proc. World Haptics 2013, pages 247-252, 2013.

[15] S. Gumtau. Tactile semiotics: the meanings of touch explored with low-tech prototypes. In Eurohaptics Conference, 2005 and Symposium on Haptic Interfaces for Virtual Environment and Teleoperator Systems, 2005. World Haptics 2005. First Joint, pages 651-652, 2005.

[16] A. Lanatà, G. Valenza, and E. Scilingo. A novel eda glove based on textile-integrated electrodes for affective computing. Medical and Biological Engineering and Computing, 50:1163-1172, 2012

[17] A. Mehrabian and J. A. Russell. An approach to environmental psychology. the MIT Press, 1974

[18] J. Posner, J. Russell, and B. Peterson. The circumplex model of affect: An integrative approach to affective neuroscience, cognitive development, and psychopathology. Development and Psychopathology, 17(03):715-734, 2005.

[19] J. L. Rhudy, A. E. Williams, K. M. McCabe, J. L. Russell, and L. J. Maynard. Emotional control of nociceptive reactions (econ): do affective valence and arousal play a role? Pain, 136(3):250-261, 2008.

[20] E. Ruiz-Padial, J. Vila, and J. F. Thayer. The effect of conscious and non-conscious presentation of biologically relevant emotion pictures on emotion modulated startle and phasic heart rate. International Journal of Psychophysiology, 79(3):341-346, 2011.

[21] K. Salminen, V. Surakka, J. Lylykangas, J. Raisamo, R. Saarinen, R. Raisamo, J. Rantala, and G. Evreinov. Emotional and behavioral responses to haptic stimulation. In Proceedings of the SIGCHI Conference on Human Factors in Computing Systems, CHI '08, pages 15551562, New York, NY, USA, 2008. ACM.

[22] A. Serio, M. Bianchi, , and A. Bicchi. A device for mimicking the contact force/contact area relationship of different materials with applications to softness rendering. In Proc. IEEE/RSJ IROS 2013, 2013.

[23] C. Swindells, K. E. MacLean, K. S. Booth, and M. Meitner. A case study of affect measurement tools for physical user interface design. In In Proc. graphics interface, pages 243-250, 2006.

[24] K. Takahashi, H. Mitsuhashi, K. Murata, S. Norieda, and K. Watanabe. Feelings of animacy and pleasantness from tactile stimulation. 2011.

[25] D. Tsetserukou. Haptihug: a novel haptic display for communication of hug over a distance. In Eurohaptics Conference 2010, pages 340 $347,2010$.

[26] G. Valenza, P. Allegrini, A. Lanatà, and E. Scilingo. Dominant lyapunov exponent and approximate entropy in heart rate variability during emotional visual elicitation. Frontiers in Neuroengineering, 5, 2012.

[27] G. Valenza, A. Lanatà, and E. Scilingo. Oscillations of heart rate and respiration synchronize during affective visual stimulation. Information Technology in Biomedicine, IEEE Transactions on, 16(4):683690, 2012.

[28] G. Valenza, A. Lanatà, and E. Scilingo. The role of nonlinear dynamics in affective valence and arousal recognition. Affective Computing, IEEE Transactions on, 3(2):237-249, 2012.

[29] G. Valenza, A. Lanata', E. P. Scilingo, and D. De Rossi. Towards a smart glove: Arousal recognition based on textile electrodermal response. In Engineering in Medicine and Biology Society (EMBC), 2010 Annual International Conference of the IEEE, pages 3598-3601. IEEE, 2010.

[30] S. Yohanan and K. MacLean. Design and assessment of the haptic creature's affect display. In In HRI '11 Proceedings of the 6th international conference on Human-robot interaction, pages 473-480, 2011.

[31] S. Yohanan and K. E. MacLean. The haptic creature project: Social human-robot interaction through affective touch. In Proceedings of the AISB 2008 Symposium on the Reign of Catz \& Dogs: The Second AISB Symposium on the Role of Virtual Creatures in a Computerised Society, volume 1, pages 7-11, 2008.

[32] S. Yohanan and K. E. MacLean. The role of affective touch in humanrobot interaction: Human intent and expectations in touching the haptic creature. International Journal of Social Robotics, 4(2):163-180, 2012.

[33] Y. Zheng and J. B. Morrell. Haptic actuator design parameters that influence affect and attention. In Haptics Symposium (HAPTICS), 2012 